\title{
Splice-tailored to fit the bug
}

One of the central features of adaptive immunity is specificity to individual microbial species. New findings reveal that insects also employ mechanisms to diversify their immune responses to microorganisms.

Dong et al. studied the role of the hypervariable pattern recognition receptor DSCAM (Down's syndrome cell adhesion molecule) in Anopheles gambiae immunity to specific pathogens. DSCAM contains ten immunoglobulin-like domains, three of which (Ig2, Ig3 and Ig7) display variability as a result of alternative splicing in their respective exon cassettes (cassettes 4 , 6 and 10). Previous work had indicated that infections with different species of pathogen correlate with distinct DSCAM splice form repertoires. So, the authors set out to characterize the contribution of immune signalling pathways and alternative splicing in DSCAMmediated species-specific responses to malaria parasite infection and to commensal gut bacteria.

Infection of mosquitoes with the rodent parasite Plasmodium berghei or with Gram-negative bacteria correlates with over-representation of DSCAM splice forms containing immunoglobulin exons 4.14 and 6.2, whereas infection with the human parasite Plasmodium falciparum increases the abundance of splice variants containing immunoglobulin exons 4.1, 6.17 and 10.21. Here, the authors showed that exons 4.1 and 6.17 are essential for the high-affinity binding of DSCAM to P. falciparum and for resistance to $P$. falciparum infection, whereas DSCAM splice forms containing exons 4.14 and 6.2 are required for control of $P$. berghei and Gram-negative commensal gut bacteria.
But how are such species-specific responses regulated? The Toll and immune deficiency (Imd) pathways are central in insect innate immunity; activation of the Toll pathway preferentially promotes $P$. berghei clearance, whereas activation of the Imd pathway constrains P. falciparum infection. Notably, the authors observed that activation of the Toll pathway correlated with a relative abundance of exon 4.14-containing DSCAM splice forms, whereas activation of the Imd pathway correlated with a relative abundance of exon 4.1-containing DSCAM splice variants. Thus, differential innate immune signalling can lead to pathogen-specific responses that involve DSCAM splice variants with increased affinities for the respective pathogen species.

Strikingly, infection with different Plasmodium species led to differential expression of putative splicing factors, including CAPER and IRSF1. For example, $P$. falciparum infection or peptidoglycanmediated Imd pathway activation led to increased transcription of CAPER and decreased transcription of IRSF1. Moreover, silencing of CAPER resulted in low levels of $P$. falciparum-responsive exon 4.1-containing DSCAM splice variants and increased susceptibility to $P$. falciparum infection, whereas IRSF1 silencing had the opposite effect. Thus, the authors suggest that nuclear factor- $\kappa \mathrm{B}$-mediated transcription downstream of the Toll and Imd pathways shapes the pathogen-specific DSCAM splice

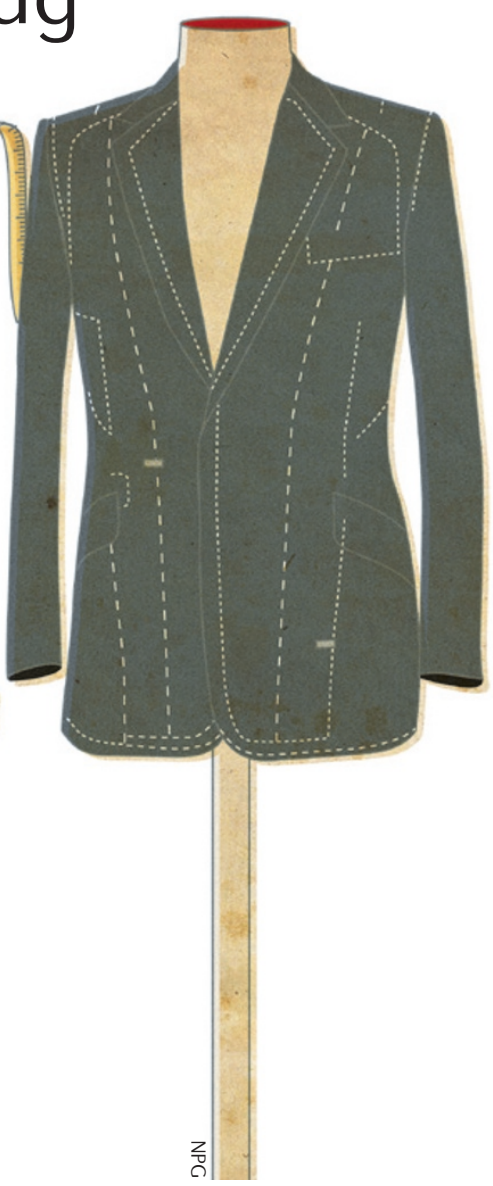

variant repertoire through CAPER, IRSF1 and potentially other splicing factors.

These findings suggest a previously unappreciated regulatory mechanism of diversified and specific immune responses in insects, and they have implications for malaria therapeutics. Maria Papatriantafyllou Locum Associate Editor, Nature Reviews Immunology

This article is modified from the original in Nature Rev. Immunol. (doi:10.1038/nri3343). pathogen-specific repertoires of the hypervariable pattern recognition receptor AgDscam. Cell Host Microbe 12, 521-530 (2012) 